International Journal of Engineering \&Technology, $7(1.7)(2018) 1-4$
SPC
International Journal of Engineering \& Technology
Website www.sciencepubco.com/index.php/IJET
Review paper

\title{
A demand for wearable devices in health care
}

\author{
K. Vijayalakshmi ${ }^{1}$, S. Uma ${ }^{1}$, R. Bhuvanya ${ }^{1}$, A. Suresh ${ }^{2}$ \\ ${ }^{1}$ Assistant Professor, Department of Computer Science and Engineering, School of Computing, Vel Tech Rangarajan Dr. Sagunthala \\ $R \& D$ Institute of Science and Technology, Avadi, Chennai-62, TamilNadu, India \\ 2 Professor \& Head, Department of Computer Science and Engineering, Nehru Institute of Engineering and Technology, T.M.Palayam, \\ Coimbatore-641105, TamilNadu, India \\ *Corresponding author E-mail: vijayalakshmi@veltechuniv.edu.in
}

\begin{abstract}
With the popularity of wearable devices, along with the development of telecommunication system there is a need for obtaining the health and fitness outcomes. So the recent advances in data analysis techniques have opened up new possibilities for using wearable technology in the digital health ecosystem. In past, it's too difficult to use the wearable devices for healthcare system because of the size of those sensors. But now with front end amplification and wireless data transmission, the wearable devices are deployed in health monitoring systems. Although the devices are continuously monitoring the human's body activity and collect various physiological data to increase the quality of human's life. In this paper first we provide a research survey on available wearable or gadgets. Also we conclude with future directions in wearable research and market.
\end{abstract}

\section{Introduction}

As the potential growth in technology, the hand held devices are available to the user such as smart phones, iPods, and tablets. The users are also having interest to wear these kinds of devices that a promise to improve the quality of human's life. But these are cannot achieve alone. These devices need to include various accessories such as garments, hats, wrist bands, socks, shoes, eyeglasses and other devices such as wristwatches, headphones, contact lenses, e-textiles and smart fabrics, headbands, beanies , jewelry such as rings, bracelets, and hearing aid-like devices that are designed to look like earrings, and skin patches are referred as wearable's. These devices are used to sense, collect and upload the physiological data in continuous manner to increasing the human's life. Some monitoring systems require the gathered sensor and wearable's data to be uploaded to a remote site such as a hospital server for further clinical analysis [1].

With the advent of cloud-computing, many wearable sensor systems can now be easily upgraded without the need for user installation of software in their monitoring devices, which makes it easier and cheaper to maintain the health monitoring system networks. Most of the present-day applications of sensors and wearable can be classified into the following five categories [2]. It's also describe about the health issues and also frequently monitoring the day to day regular body activities.

Health and Wellness Monitoring

Wearable's are monitor physiological data of all people with persistent conditions can facilitate timely medical interventions. Some user-focused health and fitness wearables are widely used by passionate peoples to gather data about their health.

Safety Monitoring

Many more number of wearable's devices has been designed for tracking the sleep, sweat, heart rate, temperature, epileptic seizures and heart attacks for older people and then send alarm signals to caregivers or emergency response teams through connected wireless devices like Bluetooth.

Home Rehabilitation

Sensing technology which is used as the combination of gaming and Virtual Reality environments and augmented feedback systems to provide home-based rehabilitation for physiotherapy, heart disease patient, and ageing individuals.

Treatment Efficiency Assessment

The availability of sensors with wearable devices can accurately measure the efficiency of patient status. They can help to track physiological changes of human, as well as the progress of treatments on a continuous basis. Sensors are also used to monitor, periodically and assess also to improve patient compliance.

Early Detection of Disorders

with combination of sensor and wearable devices we can easily identify the changes in regular activities of human's body data , this application of digital health can be used for early detection of symptoms and adverse changes in a patient's health status - helps to timely medical interventions.

\subsection{Current market and industry trends in sensors and wearable's}

The wearable devices will rise to 210 million unit shipments and $\$ 30$ billion in revenue by 2018 from 96 million unit shipments and $\$ 8.5$ billion in revenues during 2012. Note that this report includes infotainment, military and industrial markets, as well as health and fitness. The wearable Market reached 33.9 million in 2016. Another research firm, On World, predicts that in 2017, 515 million sensors for wearable, implantable or mobile health and fitness devices will be shipped globally-up from 107 million in 2012.Between 2012 and 2017, wearable health and fitness device shipments will increase by $552 \%$ and make up over $80 \%$ of the mobile sensing health and fitness device market at this time. The same firm also estimates that by 2017, 18.2 million health and 
wellness WSN systems - excluding sports/fitness devices-- will be shipped worldwide and annual revenues enabled by these systems will reach $\$ 16.3$ billion. Cloud connected services will make up 53\% of the revenues in five years. [2]

The remainder of the paper is organized as follows. In section II we survey the number of wearable devices which are helpful to monitor and predict the human's body data. In section III we survey the security issues in wearable devices. In section IV we discuss the future research work on wearable devices. In section $\mathrm{V}$ we conclude the paper.

\section{Wearable devices}

In this section, we described the recent available types of wearable devices and how they monitor and predict human's body data and help for fitness. The wearable devices are classified into major four categories. based on their application and use.

- Lifestyle and Healthcare

- E-textiles

- $\quad$ E-Patches as illustrated in fig 2.1.

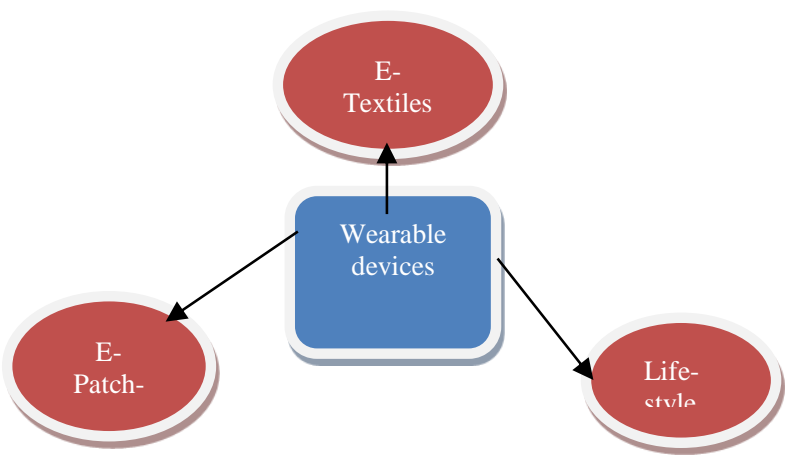

Fig. 1: Wearable Devices.

\subsection{Lifestyle and healthcare}

This kind of wearable includes sports and activity monitors such as smart band, Lark sleep sensors etc. This kind of devices is periodically monitoring the movements in sleep and other sleeping disorder. Also it's helpful to collect data on a foot. [3]

Smart Watches

Tracking performance is becoming more popular also it's important for athletes to get health data during workouts. The wearable devices are key to making such measurements. The smart watches that are used to periodically monitoring the fitness related measures such as distance run, walked, calorie consumption, heart rate, human body temperature, and quality of sleep.

Smart watches are one of the most popular wearable devices. Based on the recent Gartner reports [4], the smart watches sales are secondary when we compared with Bluetooth headsets. The functionality of the smart watches categories into two ways. First its perform some operations like mobile phone such as chatting, phone calls, weather updates. Second this device monitoring the human body activities and transfer these collected data to cloud or mobile device. In early years, in 1972 the peoples are used the first smart watch was the pulsar manufactured by Hamilton watch company. Later in 1982 the pulsar (NL C01) was released which is include user-programmable memory [5]. In recent years razor was released a dual screen smart watch which is allow us to access additional features of phone notification like phone and calls. Its also tracking the sleep movements by accelerometer [6]. If anyone who owns a band then this dual screen smart watches provide the facility to the user to exchange face book and twitter contacts by simple shaking hands.

In later the Samsung released the advanced version of Gear S2 is Samsung Gear S3 smart watch with higher specifications. It contains two types of model such as classic and LTE version Samsung Gear S3 Frontier. Both have stainless steel body, IP68 water resistance and compatible with $22 \mathrm{~mm}$ strap. Also it has 4 days battery life when its compared with Samsung Gear S2. It has built in speaker that lets you make phone calls without taking our phone from our packet. Sensors include GPS, barometer, HRM, ambient light sensor. The benefits of these sensors as, The LTE radio periodically tracking the fitness data. The speaker gives you the audio notifications also encouraging our exercises activities. It can monitor timely information as you run, and urge you on when you start to slow down. It's not very loud though, and we found it hard to make out what it was saying over the sound of our own movements and breathing.

The optical heart-rate monitor keeps tabs on your resting heart rate for entire day and delivers continuous tracking during exercise sessions. The altimeter/barometer lets you track your altitude and atmospheric pressure. There's NFC, 4GB of local storage for music - again, great for those who like to go running without their phone - and wireless charging via the WPC standard. It might stutter a little with demanding games, but when it comes to navigating the interface, checking notifications and using the watch's fitness features it's impeccably responsive [7].

In 2017, the consumer electronics show included casio WSD-F20, Misfit wearable vapor and the Garmin 5 series was released [8]. It contains GPS radio which is used to measure the accurate run rate and heart rate. Finally, we can summarize the smart watch as the successful market product for monitoring human's body activities for the entire day.

Wrist Bands

This is little similar to smart watches which is referred to bracelet like band. It has many types such as silicone wristbands, UV ultraviolet wristbands. These kind of wrist band is designed to track the activities of single person. In the early-to-mid-2000s (decade), bracelets often made of silicone became popular. They are worn to demonstrate the wearer's support of a cause or charitable organization, similar to awareness ribbons. Such wristbands are sometimes called awareness bracelets. In early 2007 they became an increasingly popular item being sold as merchandise at concerts \& sporting events worldwide. A silicone wristband is also referred as gel wristbands, jelly wristbands, rubber wristbands and fundraising wristbands [14]. All of these wristbands are made from the same silicone material.

The Medical wrist band or medical identification tape by Wenqian Qi, Yi Zhai which is mainly designed to monitor the basic medical reports of patient and help to identify the status of patient regularly [11]. It contains the hardware sensor which is connected to remote computer for monitoring the changes of patient's condition. This sensor is also reduced the nursing workload.

The activity tracking system by Lu Bai which is used to detecting the player's activities within sports. Through the inbuilt sensor of wristband and Smartphones, the tracking system can capture the game attempt of each player and provide performance analysis report about players [10]. Based on the classifier available in this tracking system we can achieve the accuracy of attempt of player in every games.

The E Lock-Holmes device by Prabhakar T.V; Madhuri Sheethala Iyer [12] is mainly designed with wrist band for measuring the changes in skin conductance level caused neural activity. Also the body media in USA has developed a disposable activity tracker which is used to measure a employee fitness periodically. Finally we can summarize the wrist band one of the most popular wearable device for monitoring sport and human's regular activities.

\subsection{E-textiles}

Its also called as smart garments, smart clothing, smart textiles, or smart fabrics that are contains digital components like battery and light including computers. Smart textiles divided into two categories aesthetic and performance enhancing. It contains light up and fabrics that are changing the colors. The performance enhancing textiles is mainly designed for sports and military applications. These contains the following advantages that are, regulating body 
temperature, reduce wind resistance, and control muscle vibrations [15].

For example, number of sensors like electromyography, heart rate and respitary sensors which are attached to fitness wear like shirts, pants, shorts, then the collected data are transmitted to smart phones. The another example is fitness pants can measure the pulse at hips, knees and ankles. Also this is connected with phone via Bluetooth for getting additional feedback about movements [16]. The GPS sports watch maker designed a polar team pro shirt mainly for athletes. It contains GPS sensor which is located on the front and back pocket and its measure the heart rate in real time. also we can able to get live training data of athletes and coaches like condition and fitness level. The lumo lift posture tracker designed a smart shorts for running. It contains the sensor can monitor the ground contact time, heart rate, stride length. We can get these kind od data through headphones also its reduces the chance of injury.

The Komodo technologies is offer a smart sleeve which is used to monitor a heart rate data, monitor a sleep, workout intensity, and body temperature. Also its help to detect heart diseases, stress level [16]. Finally we can summarize the E-textiles helps the people to prevent them from heart disease and make themselves to be fit in health.

\subsection{E-patches}

E-patches is placed on to the skin to monitor specific dose of medication through the skin. In sport, exercise and healthcare system, there is a need to predict human activities. In 2016, the E-Ink working started with LTS, a German company that makes traditional patches for smoking cessation. It's a 2-inch coin shaped battery which is used to patient performance. The Qualcomm life's tracking ambition designed a patch mainly for measuring the biometric parameters such as temperature, heart rate, and motion and gait. Then the Samsung S-patch 3 is specially designed for summer season those are measuring the heart rate, skin temperature, galvanic skin response and fat data. The HAX Accelerator which is working on the bio-sensing patch wear on chest monitors your breathing data and heart vibrations. The Binghamton university developed a paper based patch for measuring a glucose to diabetics [18].

Many road accidents happen in entire world. For reducing that the University of California san Diego developed a flexible wearable sensor along with patch which is used to predict human's body alcohol level. This device contains a temporary tattoo which is sticks on their skin. So we can easily measure the blood alcohol level from human's body sweat also easily we can collect the data through Bluetooth which is already connected with patch sensor[19].Here is the wearable multisensing patch can periodically measure the lactate in human's body sweat [17]. The wearable electronic sensor with this system can also measure the sweat metabolites, electrolytes, and temperature. This system also transmit the collected data for real time use.

The chrono therapeutics developed a smoking therapy which is to help reduce the smoking urges and its connected with for data support. For monitoring the cardiac through 3-channels was developed by DELTA company. The G-tech patch mainly designed for predicting the accurate diagnosis of gastrointestinal disorders.another example for skin patch, the Ionto team has designed a iontophoretic technology patch periodically reduce the wrinkles on the face specifically aroung the eyes. Also the Gentag produces a invisible disposable skin patches to deleiver drugs and monitoring fitness, skin care, diabetics monitoring and drug delivery for a different medicine. For monitoring the glucose level of diabetics the Nemaura Medical was developed a Sugar BEAT system. Omni pod is another drug delivery path for monitoring the insulin level of diabetics which is attached to the patient for a 3 days. The HIVOX has developed a pain relief patch which attached in various region of human's body.X2 Bio-systems is another expel for smart patch that can be placed behind the ear. This is useful for athletes to reducing their head injuries [20]. Finally we can sum- maries the smart patches are used in human's healthcare and fitness. Its mainly for periodically monitoring human's body activities like sweat, temperature, heart rate, players activities etc.

\subsection{Accessories}

\section{Smart Eyewear}

The smart eyewear is more popular head mounted devices. First the Google Glass has launched for autism children. The vuzix smart glasses promising to get emails and youtube videos and voice prediction also provide a touchpad controls. The SOLOS enabling cyclists to get real-time data including speed, cadence, heart rate and power zone. Like this the Eyesight Raptor showing mapping data, heart rate information and other information. Another example for smart eyewear is ODG's R7 AR Glasses. It has $720 \mathrm{p}$ lens that shows transparent video. The SAFILO $\mathrm{X}$ is line of line sensing glasses. Also it contains the following sensors like 3axis accelerometer, gyroscope, magnetometer, UV temperature and pressure sensors. Its helps to get exercise, brain and meditation activities. In 2017, the VUE helps to control the calls and music [16]. Finally all these kind of smart eyewear can help the people to sensing mapping information and to get other health activities information.

Smart Jewellery

The smart jewellery as a trend for the past three years. Startups like next generation of earrings, necklaces, bracelets, rings. First the ringly aries comes with $18 \mathrm{k}$ gold platted of different colors. Its helps to monitor exercise and stress. The MOTIV is a smart ring for fitness monitoring from the finger. The hardware contains titanium with $8 \mathrm{~mm}$ wide finishes of rose gold. Additionally it has the feature of getting heart rate with help of sensor inside it. The one example for tracking the step count and sleep is Misfit Swarovski shine. The upgrade device in 2017 is Fitbit Alta is very good tracker in your regular work. Also a OURA Ring designed with 6-13 inch sizes and its mainly for tracking the sleep [16]. At the end this session we summarize about the existing and recent wearable devices and its benefits in healthcare system.

\section{Security issues in wearable devices}

\subsection{Physical data access}

The fact that all kind of wearable devices are stored human's body data into local storage device without encryption. Also it doesn't provide any PIN and password protection. So the unauthorized people can easily access your personal data.

\subsection{Insecure wireless connectivity}

The wearable devices are connected with laptop, smart phone using Bluetooth, and Wi-Fi. Its possibly to get all information by brute force attack.

Its illustrated in Fig 3.1. In following figure the human's body activities data periodically transmitted to the local devices through the Bluetooth without using the any encryption method. It causes security threats to privacy data.

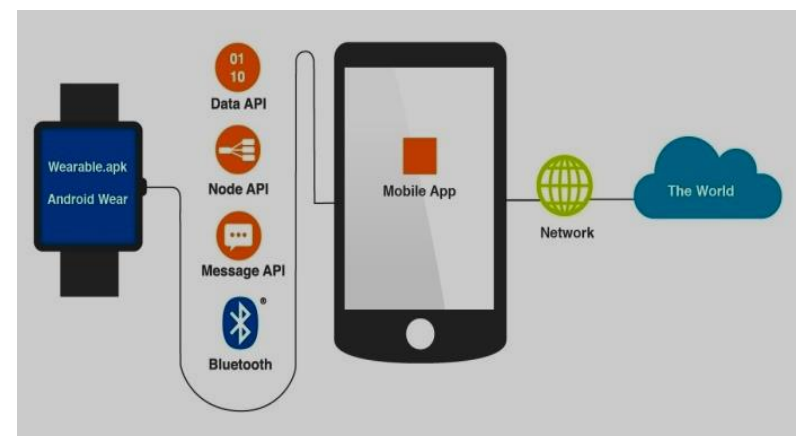

Fig. 2: Communication between Wearable Devices and Local Device via Bluetooth. 


\subsection{Sensitive data exposure}

The wearable devices that are like smart watches, wrist band contains all kind of information about users. They have been stored directly. With this the lacking of encryption they could easily access all sensitive data like bank account details, social security number, personal body information by hackers.

\subsection{Medical failure}

Based on smart wearable device data the doctor can monitor and get human's health information without visit their patient regularly. But this open opportunity to the attacker can remotely shutdown the pacemaker that causes the heart failure immediately.

\section{Challenges and research development on wearable devices}

The growth of the wearable technology increased to 68.7 million high in 2019 and this posed significant change in enterprises and lifestyle of human beings. Wearable are also fulfilling the fields of medical and safety. The E-Textile sale expected to reach 20 million units by 2020 . Also by 2021 Gartner that estimate the sale of smart watches to reach $17 \%$ more compared to now. By 2020, the smart eye wear expected to reach $40 \%$ extra in sale. It's illustrated in the comparison table 4.1

Table 1: Forecast for Wearable Devices Worldwide

\begin{tabular}{|c|c|c|c|c|}
\hline Devices & 2016 & 2017 & 2018 & 2021 \\
\hline Smart watch & 34.80 & 41.50 & 48.20 & 80.96 \\
\hline Wristband & 34.97 & 44.10 & 48.84 & 63.86 \\
\hline Sports Watch & 21.23 & 21.43 & 21.65 & 22.31 \\
\hline Bluetooth headset & 128.50 & 150.00 & 168.00 & 206.00 \\
\hline
\end{tabular}

The global wearable devices market continues to gain rapid adoption of these devices among patients for their medication, status report. In wearable devices the key issues challenging the market includes the privacy concerns like transmitting privacy data about patient condition and devices battery life. In order to maintain the security of these privacy data need to achieve device code and regulations. Also the management of privacy data is the future challenges in wearable devices because unavailability of security code and PIN, password during the transmission of those data to local devices like laptop, smart phones.

\section{Conclusion}

In last few years the wearable devices are most popular in day to day life. In this paper we surveyed regarding various kind of wearable products and its medical benefits. Also we surveyed possible security issues in these kinds of wearable devices. Finally we summarized the research development in market place and future challenges on wearable device in medical field.

\section{Acknowledgement}

We thank everyone who has provided insight and expertise that greatly assisted the research survey. We are also immensely grateful to the editorial board for their comments on early version of the manuscript.

\section{References} http://www.wearabledevices.com/, 2014.

[2] https://innovatemedtec.com/, 2016.
[3] Saewon Kye, Taeho Lee, Sangyeop Lee, Kyoungwoo Lee, Seung-chul Shin, Yong Seung Lee, "Detecting periodic limb movements in sleep using motion sensor embedded wearable band", in 2017 IEEE conference on Systems, Man and cybernetics(SMC)

[4] Gartner Inc., "Gartner says worldwide wearable devices sales to grow 18.4 percent in 2016," http://www.gartner.com, 2016.

[5] Doensen, Pieter. "Q.5 Watches with Memory and Database". WATCH. History of the modern wrist watch. Pieter Doensen. Retrieved 17 September 2010.

[6] "Razer Nabu Watch is a dual screen smartwatch with year-long battery life". Archivedfrom the original on 6 June 2016.

[7] "Samsung Gear S3 Classic and Frontier Unveiled - SmartWatch Specifications". 1 September 2016. Archived from the original on 7 September 2016. Retrieved 1 September 2016.

[8] "Paul Lamkin", https://www.forbes.com, 2017

[9] "Wallace Chu ", https://www.wristbands.com, 2013

[10] Lu Bai, Christos Efstratiou , Chee Siang Ang ,"weSport: Utilising Wrist-Band Sensing to Detect Player Activities in Basketball Games ", The Second IEEE International Workshop on Sensing Systems and Applications Using Wrist Worn Smart Devices, 2016.

[11] Wenqian Qi, Yi Zhai," The Study on the Life Signs of Clinical Patients Monitored by Electronic Wrist Band", IEEE International Conference, Computational Science and Engineering (CSE) and Embedded and Ubiquitous Computing (EUC), 2017.

[12] Prabhakar T.V; Madhuri Sheethala Iyer; Jamadagni H.S ; Priyanka P R ; Payal Mondal ; V V S Sasi Kiran ; Vaishnavi Govindarajan,” Wearable Device for Health Care Applications", India Educators' Conference (TIIEC), Texas Instruments, 2013

[13] Akicic, JM; Davis, KK; Rogers, RJ; King, WC; Marcus, MD; Helsel, D; Rickman, AD; Wahed, AS; Belle, SH (20 September 2016). "Effect of Wearable Technology Combined With a Lifestyle Intervention on Long-term Weight Loss: The IDEA Randomized al". JAMA. 316 (11):11611171. PMID 27654602. https://doi.org/10.1001/jama.2016.12858.

[14] Mulligan, Sean (9 September 2015). "SiliconeWristbandsExplained". Wristband Bros. Sean Mulligan. Retrieved 9 September 2015.

[15] Applications of Smart and Interactive Textiles". Textile Learner. Saddamhusen Jamadar. Archived from the original on 2013-0612. Retrieved 2013-04-21.

[16] MichaelSawh,"https://www.wareable.com”,2017.

[17] S. Anastasova, B. Crewther, P. Bembnowicz, V. Curto, H.M. Ip, B. Rosa, G.-Z. Yang Corrigendum to "A wearable multisensing patch for continuous sweat monitoring" [Biosens. Bioelectron. (2016)] Biosensors and Bioelectronics, Volume 94, 15 August 2017. https://doi.org/10.1016/j.bios.2017.03.018.

[18] Andrew Williams,"www.wearable.com,2017

[19] UC San Diego,"Flexible Wearable electronic skin patch to monitor alcohol levels", 2016.

[20] Ayliffe Brown," http://www.wearable-technologies.com", 2017

[21] Michelle Drolet,"https://www.csoonline.com, 2016. 